\title{
EVALUACIÓN DE DOSIS DE NITRÓGENO Y FÓSFORO EN EL CULTIVO DE PITAHAYA (Hylocereus undatus) ${ }^{1}$
}

\author{
Oscar López Turcios ${ }^{2}$; Alfonso Guido Miranda ${ }^{3}$
}

\begin{abstract}
RESUMEN
Evaluación de dosis de nitrógeno y fósforo en el cultivo de pitahaya (Hylocereus undatus). En 1992 se estableció un experimento con pitahaya en el Centro Experimental Campos Azules, en un suelo Inseptisol durante cuatro años. Los tratamientos estudiados consistieron en cuatro dosis de nitrógeno $(0 ; 40 ; 80$ y $120 \mathrm{~kg} / \mathrm{ha})$ y tres de fósforo $(0 ; 20$ y 40 $\mathrm{kg} \mathrm{P}_{2} \mathrm{O}_{5} / \mathrm{ha}$ ) combinados en un arreglo factorial (4x3), se incluyeron dos tratamientos exploratorios, potasio $(10 \mathrm{~kg}$ $\mathrm{k}_{2} \mathrm{O} / \mathrm{ha}$ ) y micronutrimentos ( $5 \mathrm{~kg} \mathrm{~B} / \mathrm{ha}, 10 \mathrm{~kg} \mathrm{Cu} / \mathrm{ha}, 10 \mathrm{Zn} / \mathrm{ha}$ y $5 \mathrm{~kg} \mathrm{Mn} / \mathrm{ha}$ ) combinados en sola mezcla con los niveles mayores de $\mathrm{N}$ y $\mathrm{P}$, totalizando 14 tratamientos, los que fueron distribuidos en bloques completos al azar con cuatro repeticiones. El nitrógeno tuvo efecto significativo en las cantidad de frutas por planta y rendimientos total en las tres cosechas (1994,1995 y 1996); el fósforo en la primera y segunda cosecha, el efecto de potasio y micronutrimentos fue significativo únicamente el la tercer cosecha. En el rendimiento, con base en la respuesta al nitrógeno, en las tres cosechas, se observó un efecto significativo en comparación al testigo $(0 \mathrm{~kg} / \mathrm{ha})$ en los niveles de 40 y $80 \mathrm{~kg} / \mathrm{ha}$ en la primera y segunda cosecha y $40 \mathrm{~kg} / \mathrm{ha}$ para la tercer cosecha y del fósforo en 20 y 40 $\mathrm{kgP}_{2} \mathrm{O}_{5}$ /ha en comparación al testigo, en la tercer cosecha todos los niveles se mostraron iguales. Al nivel económico, el nivel de $80 \mathrm{~kg}$./ha de nitrógeno resulto más rentable para la primer cosecha, con una tasa de retorno marginal de $4,46 \%$ y para la segunda y tercer cosecha el nivel de $40 \mathrm{~kg} / \mathrm{ha}$ con una tasa de retorno marginal de $41,78 \%$ y $143,43 \%$ respectivamente. Para fósforo la mayor tasa de retorno se obtuvo en el nivel $20 \mathrm{~kg} \mathrm{P}_{2} \mathrm{O}_{5} /$ ha para las tres cosechas, $10,31 \% ; 22,19 \%$ y $73,23 \%$. La tasa de retorno para potasio y micronutrimentos fue negativa en las dos primeras cosechas, sin embargo en la tercera se obtuvo una tasa de $333,76 \%$ y $129,71 \%$ para potasio y micronutrimentos respectivamente.
\end{abstract}

\begin{abstract}
Evaluation of phosphorus and nitrogen dosages in strawberry pear (Hylocereus undatus) cultivation. An experiment with strawberry pear was carried out in the Bluefields experimental center in an inseptisol soil. The experiment started in 1992, and lasted four years. The treatments studied consisted of four Nitrogen levels $(0 ; 40$; 80; and $120 \mathrm{~kg} / \mathrm{ha})$, and three Phosphorus levels (0; 20; and $\left.40 \mathrm{~kg} \mathrm{P} \mathrm{P}_{5} / \mathrm{ha}\right)$, combined in a factorial arrangement $(4 \times 3)$. Two exploratory treatments were included: Potasium $(10 \mathrm{~kg}$. $20 \mathrm{~kg} / \mathrm{ha}$ ), and rnicronutrients $(5 \mathrm{~kg} \mathrm{~B} / \mathrm{ha}, 10 \mathrm{~kg} \mathrm{Cu} / \mathrm{ha}, 10$ $\mathrm{Zn} / \mathrm{ha}$, and $5 \mathrm{~kg} \mathrm{Mn} / \mathrm{ha}$ ), combined in a single mix with the highest levels of $\mathrm{N}$ and $\mathrm{P}$. In total, there were 14 treatments, which were distributed in complete blocks at random, with four repetitions. Nitrogen had a significant effect on the amount of fruit per plant, as well as on the total yield in the three crops (1994, 1995, and 1996); Phosphorus was significant only in the first and second crops; while the effect of Potasium and the micronutrients was significant only in the third crop. Basedon the response of yield to nitrogen, the three crops showed a significant effect compared to the control $(0 \mathrm{~kg} / \mathrm{ha})$ at levels of 40 and $80 \mathrm{~kg} / \mathrm{ha}$ in the first and second crops, and $40 \mathrm{~kg} / \mathrm{ha}$ in the third crop. Concerning Phosphorus, in 20 and $40 \mathrm{~kg} \mathrm{P}_{2} \mathrm{O}_{5} / \mathrm{ha}$ as compared to the control, in the third crop all the levels were equal. In economic terms, the leve 1 of $80 \mathrm{~kg} / \mathrm{ha}$ of Nitrogen turned out to be more cost-effective for the first crop, with $41.78 \%$ and $143.43 \%$ marginal return rates, respectively. As for Phosphorus, the greatest return was obtained at $20 \mathrm{~kg} \mathrm{P}_{2} \mathrm{O}_{5}$ /ha for the three crops: $10.31 \% ; 22.19 \%$; and $73.23 \%$. The rate of return for Potasium and rnicronutrients was negative in the first two crops; however, in the third crop the return rates were $333.76 \%$ and $129.71 \%$ for Potasium and micronutrients, respectively.
\end{abstract}

\footnotetext{
1 Presentado en la XLIII Reunión Anual del PCCMCA. Panamá, 1997.

2 Centro Experimental Campos Azules INTA ZONAL A-2 Nicaragua.

3 Cultivos diversos INTA ZONALA-2, Nicaragua.
} 


\section{INTRODUCCIÓN}

La Pitahaya es una planta perenne, que crece de forma silvestre sobre árboles vivos, troncos secos, piedras y muros, es una planta que se ha utilizado en Nicaragua desde la época precolombina, sin embargo su proceso de domesticación había sido lento debido a la poca demanda que ésta tenía tanto en el mercado interno como internacional. Es a partir de 1986 que el cultivo comienza a desarrollarse a niveles comerciales, estableciéndose arcas más compactas y abriendo mercado internacionales.

Nicaragua es el segundo país productor, de la Pitahaya roja de pulpa roja, su principal mercado es la Comunidad Económica Europea. Ante esta oportunidad de mercado se inicia una tecnificación del cultivo principalmente en aspectos como vainas enraizadas en bolsas, sistemas de turoreo y manejo de densidades (Hansen 1994). El aspecto de fertilidad ha sido poco investigado, estudios sobre fertilización realizadas en Colombia mostraron que la Pitahaya responde mucho mejor a las aplicaciones orgánicas así como de la fertilización nitrogenada foliar (Natividad 1995).

Actualmente, la fertilización empleada en la mayoría de las plantaciones se basa en experiencias propias de los productores, sin tener un conocimiento técnico sobre la eficiencias y manejo de los fertilizantes, estos aplicaran de $100-200 \mathrm{~kg}$ de N/ha, $60-100 \mathrm{~kg} \mathrm{P}_{2} \mathrm{O}_{5} / \mathrm{ha}$ y $30-60 \mathrm{~kg} \mathrm{~K} 20 /$ ha, dependiendo del nivel económico del productor y no de los requerimientos nutricionales del cultivo, teniendo com consecuencias incremento en los costos de producción. Lo anterior indica que toda información obtenida en experimentos de fertilidad es de mucha importancia para el desarrollo del cultivo.

El experimento se realizó con el objetivo de conocer el efecto de dosis de nitrógeno, fósforo, potasio y micronutrimentos en los rendimientos por planta, midiendo tamaño, peso y grados brix de la fruta.

\section{MATERIALES Y MÉTODOS}

Para conocer el efecto de los fertilizantes químicos sobre el desarrollo vegetativo del cultivo, hay que disponer de datos sobre el vigor de la planta (tamaño y número de vainas),en este estudio por las características propias de manejo de la pitahaya, la que es sometida a constantes podas de formación y fitosanitarias, fue difícil evaluar esta variable, las mediciones únicamente se hicieron en los componentes de rendimiento.

El experimento se estableció en el Centro Experimental Campos Azules en Nicaragua en un suelo
Inseptisol de la serie Masatepe, alto en $\mathrm{N}$; medio en $\mathrm{P}$; alto en $\mathrm{K}, \mathrm{Ca}$ y $\mathrm{Mg}$; ligeramente ácido (Cuadro 1).

Después de ejecutado la preparación del suelo, se efectuó la plantación en junio de 1992 a una distancia de tres metros entre hilera y tres metros entre planta, para una población de 1.111 plantas/ha, usando como sostén tutores muertos de material vegetal (árboles).

El material de siembra utilizando fue el clon Rosa extraído del banco de germoplasma del mismo Centro y preparado en bolsas de polietileno con dimensiones de ocho por doce pulgadas, manteniéndose en vivero durante cuatro meses.

En este estudio se evaluaron cuatro dosis de nitrógeno $(0 ; 40 ; 80$ y $120 \mathrm{~kg} \mathrm{~N} / \mathrm{ha})$ como fuente urea $46 \%$, dos dosis de fósforo $\left(0 ; 20 ; 40 \mathrm{~kg} \mathrm{P}_{2} 0_{5} / \mathrm{ha}\right)$ y testigo; se empleó como fuente triple superfosfato $46 \% \mathrm{P} 20$ s, en arreglo factorial de doce tratamientos (Cuadro 2). Para conocer la respuesta al potasio y micronutrimentos se incluyeron dos tratamiento adicionales a los mayores niveles de N,P, uno con potasio ( $\left.10 \mathrm{~kg} \mathrm{k}_{2} \mathrm{O} / \mathrm{ha}\right)$ se usó como fuente sulfato de potasio al $52 \% \mathrm{~K}_{2} 0$ y otro con micronutrimentos $(5 \mathrm{~kg} \mathrm{~B} / \mathrm{ha}, 10 \mathrm{~kg} \mathrm{Cu} / \mathrm{ha}, 10 \mathrm{~kg} \mathrm{Zn} / \mathrm{ha}$ y $10 \mathrm{~kg} \mathrm{Mn} / \mathrm{ha}$ ) combinándose en una sola mezcla. Los 14 tratamientos se distribuyeron en bloques completos al azar con cuatro repeticiones, cada unidad experimental estuvo formada por tres plantas y el área útil por una planta con un área de $9 \mathrm{~m}^{2}$.

La fertilización empleada fue la siguiente:

Durante el primer año la aplicación de nitrógeno se fraccionó en tres épocas: $20 \%$ a la siembra en el fondo del hoyo, $40 \%$ a los 60 días y $40 \%$ a los 120 días después de la siembra, a partir del segundo año se fraccionó en dos épocas, $50 \%$ en junio y $50 \%$ en octubre.

El fósforo y potasio se fraccionó en dos épocas: para el primer año $50 \%$ a la siembra y $50 \%$ en septiembre, para los siguientes años se aplicó ell $00 \%$ en junio. Los micronutrimentos no se fraccionaron, aplicándose el $100 \%$ del tratamiento en el mes de junio de cada año.

El manejo fitosanitario contra la bacteriosis (Erwinia caratovora) y antrancnosis (Colletotrichum gloesporoides) se realizó mediante podas sanitarias y aplicaciones preventivas de oxicloruro de cobre en dosis de $2,5 \mathrm{~kg} / \mathrm{ha}$ (Instituto nicaraguense de tecnología agropecuaria 1994) las malezas se controlaron manualmente con machete.

En cuanto a la poda durante el primer año se eliminaron las vainas con orientación hacia el suelo. Cuando la planta alcanzó la parte superior del tutor se 
Cuadro 1. Análisis químico de suelos. Ensayo fertilización en Pitahaya. Campos Azules, Nicaragua. 1992.

\begin{tabular}{cccccccc}
\hline \multirow{2}{*}{$\begin{array}{c}\text { Profundidad } \\
\text { (CM }\end{array}$} & $\begin{array}{c}\text { Ph } \\
\mathbf{H}_{\mathbf{2}} \mathbf{0}\end{array}$ & $\begin{array}{c}\text { M.O } \\
\boldsymbol{\%}\end{array}$ & $\begin{array}{c}\mathbf{P} \\
\mathbf{p p m}\end{array}$ & \multicolumn{3}{c}{ Meq/100 g Suelo } \\
\cline { 6 - 8 } & & $\mathbf{K}$ & $\mathbf{C a}$ & $\mathbf{M g}$ \\
\hline $0-20$ & 6,5 & 4,3 & 9 & 1,75 & 22,24 & 5,88 \\
\hline
\end{tabular}

Cuadro 2. Número de tratamientos en kilogramos de nutrimento por hectárea por año. Ensayo fertilización en Pitahaya. Campos Azules, Nicaragua. 1996.

\begin{tabular}{rcccc}
\hline No. & $\begin{array}{c}\mathbf{N} \\
\mathbf{k g} / \mathbf{h a} / \mathbf{a n ̃ o}\end{array}$ & $\begin{array}{c}\mathbf{P}_{\mathbf{2}} \mathbf{0}_{\mathbf{5}} \\
\mathbf{k g} \mathbf{h a} / \mathbf{a n ̃ o}\end{array}$ & $\begin{array}{c}\mathbf{K}_{\mathbf{2}} \mathbf{0} \\
\mathbf{k g} / \mathbf{h a} / \mathbf{a n ̃ o}\end{array}$ & $\begin{array}{c}\text { Microelemento } \\
\mathbf{k g}\end{array}$ \\
\hline 1 & 0 & 0 & 0 & 0 \\
2 & 0 & 20 & 0 & 0 \\
3 & 0 & 40 & 0 & 0 \\
4 & 40 & 0 & 0 & 0 \\
5 & 40 & 20 & 0 & 0 \\
6 & 40 & 40 & 0 & 0 \\
7 & 80 & 0 & 0 & 0 \\
8 & 80 & 20 & 0 & 0 \\
9 & 80 & 40 & 0 & 0 \\
10 & 120 & 0 & 0 & 0 \\
11 & 120 & 20 & 0 & 0 \\
12 & 120 & 40 & 0 & 0 \\
13 & 120 & 40 & 10 & Mic $(1)$ \\
14 & 120 & 40 & 0 & \\
\hline
\end{tabular}

(1): $5 \mathrm{~kg} \mathrm{~B} / \mathrm{ha} / \mathrm{año}, 10 \mathrm{~kg} \mathrm{Cu} / \mathrm{ha} /$ año y $10 \mathrm{~kg} \mathrm{ZnSo}{ }_{4} / \mathrm{ha} /$ año y $\mathrm{Mn} 5 \mathrm{~kg} / \mathrm{ha} /$ año.

realizó otra poda para estimular la formación de vainas, posteriormente se eliminaron las vainas pequeñas y entrelazada para evitar el efecto de competencia por nutrimentos y sombra (Guzmán 1996).

La duración del experimento fue de cuatro años evaluándonse tres ciclos de cosechas $(1994,1995$, y 1996). Las variables medidas fueron: largo y diámetro de fruta, peso de pulpa y cáscara, peso unitario de fruto, frutas por planta, grados brix y rendimiento, estas mediciones se hicieron en todas las frutas producidas en la planta control de cada unidad experimental.

Los datos fueron analizados a través de análisis de varianza y separación de medias por el método de Duncan. La metodología utilizada. en la evaluación económica consistió presupuesto parcial y tasa de retorno marginal (CIMMYT, 1988).

\section{RESULTADOS Y DISCUSIÓN}

El Cuadro 3 presenta el efecto del nitrógeno, fósforo, potasio y micronutrimentos en el tamaño de la fruta, frutas por planta, grados brix y rendimiento durante tres cosechas (1994, 1995 y 1996).

Tamaño de fruta: para caracterizar el tamaño de la fruta se consideraron las variables largo, diámetro y peso (pulpa y cáscara). Durante los tres años de cosecha no se obtuvieron diferencias significativas por las aplicaciones de fertilizante lo que demuestra que éste no tiene efecto sobre el tamaño y peso de la fruta. Según caracterización hecha por Maltez (1994) la fruta del clon Rosa presenta pesos promedios de 400 gramos, en este estudio el peso promedio en todos los niveles y nutrimentos fue de 390 gramos, esto quiere decir que prevaleció el efecto genético sobre el fertilizante.

Frutas por planta: En la primer cosecha se obtuvieron diferencias significativas a las aplicaciones de nitrógeno en los niveles de 80 y $120 \mathrm{~kg} / \mathrm{ha}$, la dosis de 40 $\mathrm{kg} / \mathrm{ha}$ no presentó diferencias significativas en comparación al testigo. La dosis media de fósforo $(20 \mathrm{~kg}$ $\mathrm{P}_{2} \mathrm{O}_{5} / \mathrm{ha}$ ) tuvo una influencia significativa en la producción de frutas. Los tratamientos con potasio y micronutrimentos no influyeron significativamente. 
Cuadro 3. Efecto del nitrógeno, fósforo, potasio y micronutrimentos sobre el rendimiento y sus componentes durante tres ciclos de cosecha (1994, 1995 Y 1996) Campos Azules. Nicaragua.

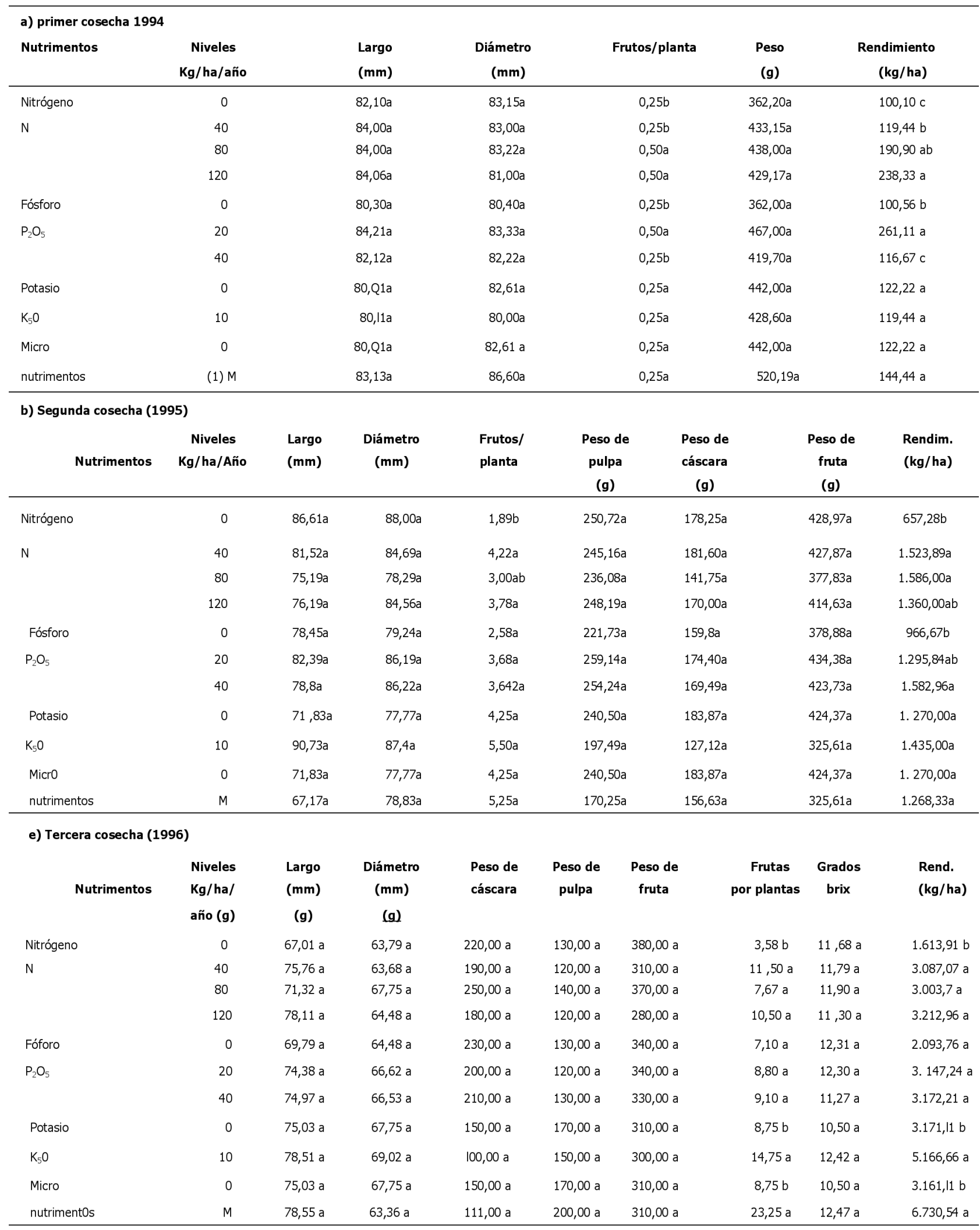

(1) $5 \mathrm{k} \mathrm{B/ha,} 10 \mathrm{~kg} \mathrm{Cu/ha/año,} 10 \mathrm{~kg}, \mathrm{Zn} / \mathrm{ha} / \mathrm{año}$ y $5 \mathrm{~kg} \mathrm{Mn} / \mathrm{ha} / \mathrm{año}$ 
Durante la segunda cosecha la aplicación de nitrógeno presentó efecto significativo obteniéndose el mayor número de frutas en el nivel de $40 \mathrm{~kg} / \mathrm{ha}$. El efecto del fósforo fue similar a la primer cosecha obteniéndose la mayor producción de frutas en los niveles de 20 y $40 \mathrm{~kg}$. $\mathrm{P}_{2} \mathrm{O}_{5} / \mathrm{ha}$. El potasio y micronutrimentos no presentó diferencias significativas. Resultados similares reporta Guzmán (1994). donde indica que el cultivo depitahaya responde a las aplicaciones de nitrógeno y fósforo y no al potasio.

Durante la tercera cosecha se mantuvo el efecto del nitrógeno y se obtuvo la mayor producción de frutas en el nivel del $40 \mathrm{~kg}$./ha, sin embargo el fósforo no ejerció un efecto significativo, esto se puede deber a que este elemento se mueve muy poco en la mayoría de los suelos, por lo general se queda en el lugar donde es puesto y nunca se acercará lo suficiente a las raíces para ser absorbido, Pereira (1991), las aplicaciones de Zn pueden limitar la disponibilidad de fósforo. En este estudio las aplicaciones se hicieron sobre la superficie del suelo a la entrada de la época lluviosa sin incorporarlo para evitar daño de raíces.

Con respecto a los tratamientos con potasio y micronutrimentos el efecto fue significativo .en la tercer cosecha, en el caso del postasio no hay causas aparentes que expliquen el aumento en la producción de frutas por planta ya que el contenido de potasio intercambiable en este suelo es de 1,75 miliequivalente por 100 gramos de suelo, por lo tanto no se puede atribuir a este nutrimento la variación en producción, según estudios realizados por el Ministerio de Agricultura y Ganadería (1992) los suelos de esta región contienen más de 0,5 me de k/100 g de suelo lo que, de acuerdo a parámetros internacionales es alto.
En este experimento no se determinaron los contenidos de micronutrimentos en el suelo, no pudiéndose correlacionar su efecto, sin embargo estudios realizados por el Ministerio de Agricultura y Ganadería (1992) no observaron efecto a las aplicaciones de micronutrimentos en los cultivos de arroz, ajonjolí,algodón, soya, sorgo, maíz, frijol, tabaco y papa. Estos resultados evidencian la necesidad de continuar haciendo estudios sobre el efecto de potasio y micronutrimentos.

La medición de los grados brix se realizó en la tercera cosecha y no se encontró efecto significativo a las aplicaciones de nitrógeno, fósforo, potasio y micronutrimentos. Los resultados muestran variaciones de 10,50\% a 12,24\%, según Cruz (1992) el contenido de grados brix en este don es de 11,4\% encontrándose dentro del rango determinado.

El rendimiento estuvo en relación directa a la producción de frutas por planta, al analizar el acumulado de las tres cosechas (Cuadro 4). Las aplicaciones de nitrógeno tuvieron mayor incremento en el nivel de 40 $\mathrm{kg} / \mathrm{ha}$ y en fósforo en $40 \mathrm{~kg}$ de $\mathrm{P}_{2} \mathrm{O}_{5} / \mathrm{ha}$, en cuanto al potasio y micronutrimentos, el incremento fue mayor en el acumulado por potasio.

El Cuadro 5 presenta las tasas de retorno marginal para las tres cosechas. Para el cálculo de presupuesto parcial se consideró un precio de $\mathrm{C} \$ 15,60$ pagado al agricultor por kilogramo de pulpa para los tres períodos, en el caso de nutrimentos se considera el precio por kilogramo para nitrógeno $\mathrm{C} \$ 17,70$, fósforo $\mathrm{C} \$ 9,57$, potasio $\mathrm{C} \$ 8,46$ y micronutrimentos $\mathrm{C} \$ 13,20$.

En el primer año de cosecha las aplicaciones de nitrógeno tuvieron un efecto lineal, la mayor tasa de

Cuadro 4. Rendimiento acumulado para tres ciclos de cosecha (kg/ha)Campos Azules. Nicaragua(1994-96).

\begin{tabular}{|c|c|c|c|c|c|}
\hline Nutrimento & Nivel & 1994 & 1995 & 1996 & $\begin{array}{c}\text { Incremento } \\
\text { kg/ha/año \% }\end{array}$ \\
\hline Nitrógeno & 0 & 100,00 & 757,28 & $2.371,19$ & \\
\hline \multirow[t]{3}{*}{$(\mathrm{N})$} & 40 & 119,44 & $1.643,33$ & $4.863,97$ & 105,12 \\
\hline & 80 & 190,9 & 1. 776,9 & $4.602,03$ & 94,08 \\
\hline & 120 & 238,33 & $1.598,33$ & $4.811,29$ & 102,91 \\
\hline Fósforo & 0 & 100,11 & $1.067,23$ & $3.160,99$ & \\
\hline \multirow[t]{2}{*}{$\mathrm{P}_{2} \mathrm{O}_{5}$} & 20 & 261,67 & $1.556,95$ & $4.704,19$ & 48,82 \\
\hline & 40 & 116,67 & $1.699,63$ & $4.871,84$ & 54,12 \\
\hline Potasio & 0 & 122,22 & $1.557,22$ & $4.718,33$ & \\
\hline $\mathrm{K}_{2} \mathrm{O}$ & 10 & 119,44 & $1.389,44$ & $6.556,10$ & 38,95 \\
\hline Micro & 0 & 122,22 & $1.557,22$ & $4.718,33$ & \\
\hline Nutrimentos & M & 144,44 & $1.412,77$ & $8.143,31$ & 72,59 \\
\hline
\end{tabular}


Cuadro 5. Tasa de retorno marginal en la evaluación de niveles de nitrógeno, fósforo, potasio y micronutrimentos en campos azules. Nicaragua (1994-96).

\begin{tabular}{lcrrr}
\hline \multirow{2}{*}{ Nutrimentos } & Niveles & \multicolumn{3}{c}{ Tasa de retorno marginal (\%) } \\
\cline { 3 - 5 } & $\mathbf{k g} / \mathbf{h a} / \mathbf{a n ̃ o}$ & $\mathbf{1 9 9 4}$ & $\mathbf{1 9 9 5}$ & $\mathbf{1 9 9 6}$ \\
\hline Nitrógeno & 40 & $-4,00$ & 41,78 & 143,43 \\
$(\mathrm{~N})$ & 80 & 1,24 & 21,87 & 68,98 \\
120 & 4,46 & 10,53 & 49,09 & \\
Fósforo & 0 & & & \\
P20s & 20 & 10,31 & 73,23 & 22,19 \\
40 & $-0,24$ & 37,99 & 20,71 & \\
Potasio & 0 & & & \\
K20 & 10 & $-1,46$ & $-28,54$ & 333,76 \\
Micro & & & & \\
Nutrimentos & $\mathrm{M}$ & $-0,19$ & $-7,1$ & 129,71 \\
\hline
\end{tabular}

retorno se obtuvo en el nivel de $120 \mathrm{~kg} / \mathrm{ha}$, para la segunda y tercer cosecha, la tasa de retorno más alta se obtuvo en el nivel $40 \mathrm{~kg} / \mathrm{ha}$, lo anterior sugiere la posibilidad de adición excesiva de urea, ya que no se necesitan grandes cantidades para obtener altos beneficios. En cuanto al fósforo, el cultivo necesitó de pocas cantidades para satisfacer las requerimientos nutricionales, en las tres cosechas la tasa más alta se mantuvo en el nivel de $20 \mathrm{~kg} \mathrm{P}_{2} \mathrm{O}_{5} / \mathrm{ha}$.

Las aplicaciones de potasio y micronutrimentos no fueron rentables para las primeras dos cosechas. Se obtuvieron tasas de retorno marginales negativas, para la tercer cosecha no hay causa aparente que explique el incremento de los rendimientos y altas tasas de retorno.

\section{CONCLUSIONES}

El tamaño, peso de la fruta y grados brix, no se vieron afectados por la aplicación de nutrimentos.

La mayor producción de pitahaya se obtuvo con las aplicaciones de nitrógeno y fósforo.

La alternativa de aplicar potasio y micronutrimentos debe ser más estudiada.

La pitahaya presentó una respuesta significativa económica a las aplicaciones de nitrógeno y fósforo.

\section{LITERATURA CITADA}

CIMMYT. 1988 La formulación de recomendaciones técnicas a partir de datos agronómicos: Un manual metodológico de evaluación económica. México. 79 p.
CRUZ P. H. 1992. Fisiología de la pitahaya. Trabajo presentado en el primer encuentro nacional del cultivo de pitahaya, San Marcos, Nicaragua. p. 14-20.

GUZMAN R. 1994. Fertilización de la pitahaya. Trabajo presentado en el primer encuentro nacional del cultivo de la pitahaya, San Marcos, Carazo Nicaragua. p.80-82.

HANSEN, J. 1994. Aspectos económicos y financieros del cultivo de Pitahaya. Trabajo presentado en el primer encuentro nacional del cultivo de Pitahaya. San Marcos, Nicaragua. 118 p.

INSTITUTO NICARAGUENSE DE TECNOLOGÍA AGROPECUARIA. 1994. Guía técnica para la producción de pitahaya, San Marcos, Carazo, Nicaragua. 52 p.

MALTEZ R. 1994. Características de las variedades de pitahaya cultivadas en Nicaragua. Trabajo presentado en el primer encuentro Nacional del cultivo de pitahaya, San Marcos. $11 \mathrm{p}$.

MINISTERIO DE AGRICULTURA Y GANADERIA. 1992a. Resultados de la fertilización con potasio en Nicaragua. MAG-FAO. Managua, Nicaragua.

MINISTERIO DE AGRICULTURA Y GANADERIA 1992b. Efecto de fertilización con azufre y micronutrimentos en Nicaragua. MAG-FAO. Managua, Nicaragua. $13 \mathrm{p}$.

NATIVIDAD R. 1995 El cultivo de pitahayas y sus perspectivas de desarrollo en México, Tabasco, México. 29 p.

PEREIRA. 1991. Aspectos fisiológicos de la productividad vegetal. Instituto de la Potasa y el Fósforo. Quito, Ecuador. 12 p. 